01

\title{
Об одной обратной задаче фазового превращения в твердых телах
}

\author{
(C) Х.М. Гамзаев
}

Азербайджанский государственный университет нефти и промышленности, AZ 1010 Баку, Азербайджан

e-mail: xan.h@rambler.ru

(Поступило в Редакцию 19 апреля 2017 г. В окончательной редакции 24 февраля 2018 г.)

Рассмотрен процесс диффузионного фазового превращения, описываемый нелинейной двухфазной моделью Стефана с подвижной границей. В рамках данной модели поставлена обратная задача, заключающаяся в определении граничного режима, обеспечивающего перемещения подвижной границы по заранее заданному закону. Для численного решения обратной задачи предложен вычислительный алгоритм, основанный на использовании метода выпрямления фронтов и вариационного подхода с локальной регуляризацией.

DOI: 10.21883/JTF.2018.08.46299.2299

\section{Введение}

Известно, что в твердых телах могут реализоваться фазовые превращения двух типов: бездиффузионные, происходящие без изменения химического состава и не связанные с диффузионным перераспределением компонентов, и диффузионные, при которых лимитирующим звеном процесса является перенос примеси [1-4]. Часто для моделирования процессов диффузионного фазового превращения в твердых телах пользуются моделью Стефана с двухфазной зоной $[1,2]$. При использовании модели Стефана с двухфазной зоной математическая постановка задачи сводится к двум дифференциальным уравнениям в частных производных с соответствующими краевыми условиями, описывающими диффузии в первой и во второй фазах, и уравнению материального баланса на границе раздела фаз. Решение этой задачи, т.е. нахождение распределения концентрации растворенного вещества в фазах и положения границы раздела фаз, осуществляется в основном аналитическими методами для случая постоянства коэффициентов диффузии, граничных и начальных условий. Однако анализ реальных процессов фазового превращения показывает, что условия протекания этих процессов зачастую таковыми не являются. Следовательно, применение результатов таких аналитических исследований может привести к получению неверных оценок параметров диффузионного фазового превращения.

Необходимо отметить, что эффективность процессов фазового превращения во многом зависит от закона перемещения подвижной границы раздела фаз. В связи с этим очень важным является задача регулирования движения подвижной границы раздела фаз в диффузионных фазовых превращениях. В настоящей работе проблема регулирования движения границы раздела двух фаз представляется как граничная обратная задача для системы уравнений диффузионного фазового превращения.

\section{Постановка задачи}

Пусть рассматривается процесс диффузионного фазового превращения в конечной области, описываемый моделью Стефана с двухфазной зоной:

$$
\begin{aligned}
\frac{\partial c_{1}}{\partial t} & =\frac{\partial}{\partial x}\left(D_{1}\left(c_{1}\right) \frac{\partial c_{1}}{\partial x}\right), \\
(x, t) \in \Omega^{-} & =\{0<x<s(t), 0<t \leq T\}, \\
\frac{\partial c_{2}}{\partial t} & =\frac{\partial}{\partial x}\left(D_{2}\left(c_{2}\right) \frac{\partial c_{2}}{\partial x}\right), \\
(x, t) \in \Omega^{+} & =\{s(t)<x<L, 0<t \leq T\},
\end{aligned}
$$

где $c_{i}(x, t), D_{i}\left(c_{i}\right), i=1,2$ - концентрация и коэффициент диффузии растворенного вещества в соответствующей фазе, $s(t)$ - координата подвижной границы раздела фаз.

Пусть в начальный момент времени $t=0$ распределение концентрации растворенного вещества в фазах и положение границы раздела фаз известны, т.е. для системы (1), (2) имеем следующие начальные условия:

$$
c_{1}(x, 0)=\phi_{1}(x), \quad c_{2}(x, 0)=\phi_{2}(x), \quad s(0)=0 .
$$

Предположим, что в первой фазе изменение концентрации во времени на границе $x=0$ описывается функцией $f(t)$, а во второй фазе концентрация на границе $x=l$ меняется со временем по закону $\xi(t)$. Тогда на фиксированных границах $x=0$ и $x=l$ будем иметь следующие граничные условия:

$$
c_{1}(0, t)=f(t), \quad c_{2}(l, t)=\xi(t) .
$$

На границе раздела фаз $x=s(t)$ концентрация растворенного вещества равна равновесной [2]

$$
c_{1}(s(t), t)=c_{1}^{r}(t), \quad c_{2}(s(t), t)=c_{2}^{r}(t) .
$$

А закон перемещения подвижной границы представляется в виде уравнения массового баланса на границе 
раздела фаз

$$
\begin{gathered}
c_{2}(s(t), t)-c_{1}(s(t), t) \frac{d s}{d t}=D_{1}\left(c_{1}(s(t), t)\right) \frac{\partial c_{1}(s(t), t)}{\partial x} \\
-D_{2}\left(c_{2}(s(t), t)\right) \frac{\partial c_{2}(s(t), t)}{\partial x} .
\end{gathered}
$$

Следует отметить, что прямая задача диффузионного фазового превращения состоит в нахождении функций $c_{1}(x, t), c_{2}(x, t), s(t)$, удовлетворяющих уравнениям (1), (2), (6) и дополнительно заданным условиям (3)-(5). Существенной особенностью прямой задачи является наличие подвижной границы раздела фаз, закон перемещения которого определяется в ходе решения задачи $[5,6]$. Однако для процессов диффузионных фазовых превращений очень важное практическое значение имеют задачи, в которых по заранее заданному закону движения границы раздела фаз исследуются те условия для первой фазы на границе $x=0$, при которых такие движения возможны. В рамках модели (1)-(6) поставим следующую задачу: для первой фазы найти такой закон изменения во времени концентрации растворенного вещества на границе $x=0$, который обеспечивал бы перемещения границы раздела фаз по заданному закону.

Таким образом, закон перемещения границы раздела фаз $s(t)$ считается известным, и требуется определить функции $f(t), c_{1}(x, t), c_{2}(x, t)$ из уравнения $(1),(2)$ и дополнительных условий (3)-(6).

\section{Метод решения}

Используя метод выпрямления фронтов, преобразуем задачу (1)-(6). Введем следующую замену переменных:

$$
y=\frac{x-s(t)}{l-s(t)}, \quad t=t,
$$

и область задания уравнения (2) $\Omega^{+}$отобразим на область $\Omega=\{0<y<1,0<t \leq T\}$. Тогда уравнение (2) и соответствующие ему дополнительные условия запишутся в виде

$$
\begin{aligned}
& \frac{\partial c_{2}}{\partial t}=d(t) \frac{\partial}{\partial y}\left(D_{2}\left(c_{2}\right) \frac{\partial c_{2}}{\partial y}\right)+u(y, t) \frac{\partial c_{2}}{\partial y}, \\
&(y, t) \in \Omega=\{0<y<1,0<t \leq T\}, \\
& c_{2}(y, 0)=\phi_{2}(y), \\
& c_{2}(0, t)=c_{2}^{r}(t), \\
& c_{2}(1, t)=\xi(t),
\end{aligned}
$$

где $d(t)=\frac{1}{(l-s(t))^{2}} ; u(y, t)=\frac{1-y}{l-s(t)} \frac{d s}{d t}$.

Аналогичным образом замена переменных

$$
y=\frac{x}{s(t)}, \quad t=t
$$

отображает область задания уравнения (1) $\Omega^{-}$на область $\Omega$. В результате уравнение (1) и соответствующие ему дополнительные условия запишутся в виде

$$
\begin{gathered}
\frac{\partial c_{1}}{\partial t}=r(t) \frac{\partial}{\partial y}\left(D_{1}\left(c_{1}\right) \frac{\partial c_{1}}{\partial y}\right)+q(y, t) \frac{\partial c_{1}}{\partial y}, \\
(y, t) \in \Omega=\{0<y<1,0<t \leq T\}, \\
c_{1}(y, 0)=\phi_{1}(y), \\
c_{1}(0, t)=f(t), \\
D_{1}\left(c_{1}(1, t)\right) \frac{\partial c_{1}(1, t)}{\partial y}+p(t) c_{1}(1, t)=p(t) c_{2}(0, t) \\
+\lambda(t) D_{2}\left(c_{2}(0, t)\right) \frac{\partial c_{2}(0, t)}{\partial y}, \\
c_{1}(1, t)=c_{1}^{r}(t),
\end{gathered}
$$

где $r(t)=\frac{1}{s^{2}(t)} ; \quad q(y, t)=\frac{y}{s(t)} \frac{d s}{d t} ; \quad p(t)=s(t) \frac{d s}{d t} ; \lambda(t)=$ $=\frac{s(t)}{l-s(t)}$.

Таким образом, в результате применения метода выпрямления фронтов задача (1)-(6) расщепляется на две задачи, причем каждая задача рассматривается в прямоугольной области $\Omega$ с фиксированными границами. Функции $s(t), d(t), u(y, t), c_{2}^{0}(t), c_{2}^{r}(t), \xi(t)$ считаются заданными, поэтому краевая задача (7)-(10) принадлежит к классу прямых задач. Решив прямую задачу (7)-(10), можно определить $c_{2}(y, t)$. А в задаче (11)-(15) известными являются $r(t), q(y, t), \lambda(t), p(t)$, а также $c_{2}(y, t)$ из решения прямой задачи (7)-(10). Неизвестными считаются функции $c_{1}(y, t)$ и $f(t)$. Следовательно, задача (11)-(15) относится к классу граничных обратных задач [7-9].

Для численного решения задачи (7)-(10) используем метод конечных разностей. Введем равномерную разностную сетку в области $\bar{\Omega}$

$$
\begin{aligned}
\varpi= & \left\{\left(y_{i}, t_{j}\right): y_{i}=i \Delta y, \quad t_{j}=j \Delta t, \quad i=0,1,2, \ldots, n,\right. \\
& i=0,1,2, \ldots, m\}
\end{aligned}
$$

с шагами $\Delta y=1 / n$ по переменной $y$ и $\Delta t=T / m$ по времени $t$. Дискретный аналог задачи (7) $-(10)$ на сетке запишем в виде неявной разностной схемы с нелинейностью на предыдущем временном слое

$$
\begin{gathered}
\frac{c_{2, i}^{j}-c_{2, i}^{j-1}}{\Delta t}=d^{j} \frac{1}{\Delta y}\left[D_{2, i+1 / 2}^{j-1} \frac{c_{2, i+1}^{j}-c_{2, i}^{j}}{\Delta y}\right. \\
\left.-D_{2, i-1 / 2}^{j-1} \frac{c_{2, i}^{j}-c_{2, i-1}^{j}}{\Delta y}\right]+u_{i}^{j} \frac{c_{2, i+1}^{j}-c_{2, i}^{j}}{\Delta y} \\
i=\overline{1, n-1}, \quad j=\overline{1, m} \\
c_{2, i}^{0}=\phi_{2, j}, \quad i=\overline{0, n} \\
c_{2,0}^{j}=c_{2}^{r, j} \\
c_{2, n}^{j}=\xi^{j}
\end{gathered}
$$


где $c_{2, i}^{j} \approx c_{2}\left(y_{i}, t_{j}\right), D_{2, i+1 / 2}^{j-1} \approx D_{2}\left(c_{2}\left(y_{i \pm 1 / 2}, t_{j-1}\right)\right), u_{i}^{j}=$ $=u\left(y_{i}, t_{j}\right), \quad \phi_{2, i}=\phi\left(y_{i}\right), \quad c_{2}^{r, j}=c_{2}^{r}\left(t_{j}\right), \quad \xi^{j}=\xi\left(t_{j}\right)$, $d^{j}=d\left(t_{j}\right)$.

Дискретная задача (16) при каждом фиксированном значении $j, j=\overline{1, m}$ представляет собой систему линейных алгебраических уравнений с трехдиагональной матрицей, в которой в качестве неизвестных выступают приближенные значения искомой функций $c_{2}(y, t)$ в узлах разностной сетки, т. е. $c_{2, i}^{j}, i=\overline{0, n}, j=\overline{0, m}$. Для решения таких систем можно использовать алгоритм Томаса (метод прогонки) [8].

Определив численное решение задачи (7)-(10), можно перейти к численному решению задачи (11)-(15). Сначала дискретизируем уравнение (11) по переменной $t$. Производную $\frac{\partial c_{1}(y, t)}{\partial t}$ в уравнении (11) при $t_{j}$, $j=\overline{1, m}$ аппроксимируем разностью „назад““

$$
\left.\frac{\partial c_{1}(y, t)}{\partial t}\right|_{t=t_{j}} \approx \frac{c_{1}\left(y, t_{j}\right)-c_{1}\left(y, t_{j-1}\right)}{\Delta t} .
$$

Вводя обозначение $c_{1}^{j}(y) \approx c_{1}\left(y, t_{j}\right)$, уравнение (11) и условия (12)-(15) запишем в виде

$$
\begin{gathered}
\frac{c_{1}^{j}(y)-c_{1}^{j-1}(y)}{\Delta t}=r^{j} \frac{d}{d y}\left(D_{1}\left(c_{1}^{j-1}(y)\right) \frac{d c_{1}^{j}(y)}{d y}\right) \\
+q^{j}(y) \frac{d c_{1}^{j}(y)}{d y}, \quad 0<y<1 \\
c_{1}^{j}(0)=f^{j} \\
D_{1}\left(c_{1}^{j-1}(1)\right) \frac{\partial c_{1}^{j}(1)}{\partial y}+p^{j} c_{1}^{j}(1)=p^{j} c_{2}^{j}(0) \\
+\lambda^{j} D_{2}\left(c_{2}^{j}(0)\right) \frac{\partial c_{2}^{j}(0)}{\partial y} \\
c_{1}^{j}(1)=c_{1}^{r, j} \\
j=\overline{1, m} \\
c_{1}^{0}(y)=\phi_{1}(y)
\end{gathered}
$$

где $q^{j}=q\left(t_{j}\right), c_{1}^{r, j}=c_{1}^{r}\left(t_{j}\right), q^{j}(y)=q\left(y, t_{j}\right), f^{j} \approx f\left(t_{j}\right)$, $p^{j}=p\left(t_{j}\right), r^{j}=r\left(t_{j}\right), \lambda^{j}=\lambda\left(t_{j}\right)$. Теперь поставленную обратную задачу для полудискретного уравнения (17) сформулируем как вариационную задачу с использованием локальной регуляризации [8]. Для этого в соответствии с (20) введем сглаживающий функционал в виде

$$
J\left(f^{j}\right)=\left[c_{1}^{j}(1)-c_{1}^{r, j}\right]^{2}+\gamma\left(f^{j}\right)^{2},
$$

где $\gamma$ - параметр регуляризации.

Таким образом, задача определения $f^{j}$ на каждом временном слое $j=1,2, \ldots, m$ сводится к минимизации сглаживающего функционала (22) при выполнении условий (17)-(19), (21).
Предположим, что решение дифференциальноразностной задачи (17)-(19) на каждом временном слое $j=1,2, \ldots, m$ можно представить в виде $[8,10]$

$$
c_{1}^{j}(y)=w^{j}(y)+f^{j} z^{j}(y),
$$

где $w^{j}(y), z^{j}(y), j=\overline{1, m}-$ неизвестные функции. Подставив соотношение (23) в уравнение (17), будем иметь

$$
\begin{aligned}
& \frac{w^{j}(y)+f^{j} z^{j}(y)-c_{1}^{j-1}(y)}{\Delta t}=r^{j} \frac{d}{d y}\left(D_{1}\left(c_{1}^{j-1}(y)\right) \frac{d w^{j}(y)}{d y}\right) \\
& +q^{j}(y) \frac{d w^{j}(y)}{d y}+f^{j} r^{j} \frac{d}{d y}\left(D_{1}\left(c_{1}^{j-1}(y)\right) \frac{d z^{j}(y)}{d y}\right) \\
& +f^{j} q^{j}(y) \frac{d z^{j}(y)}{d y}
\end{aligned}
$$

или

$$
\begin{aligned}
& {\left[\frac{w^{j}(y)-c_{1}^{j-1}(y)}{\Delta t}-r^{j} \frac{d}{d y}\left(D_{1}\left(c_{1}^{j-1}(y)\right) \frac{d w^{j}(y)}{d y}\right)\right.} \\
& \left.-q^{j}(y) \frac{d w^{j}(y)}{d y}\right]+f^{j}\left[\frac{z^{j}(y)}{\Delta t}-r^{j}\right. \\
& \left.\times \frac{d}{d y}\left(D_{1}\left(c_{1}^{j-1}(y)\right) \frac{d z^{j}(y)}{d y}\right)-q^{j}(y) \frac{d z^{j}(y)}{d y}\right]=0 .
\end{aligned}
$$

Соотношение (23) также подставим в (18), (19)

$$
\begin{gathered}
w^{j}(0)+f^{j} z^{j}(0)=f^{j}, \\
D_{1}\left(c_{1}^{j-1}(1)\right) \frac{\partial w^{j}(1)}{\partial y}+f^{j} D_{1}\left(c_{1}^{j-1}(1)\right) \frac{\partial z^{j}(1)}{\partial y} \\
+p^{j} w^{j}(1)+f^{j} p^{j} z^{j}(1)=p^{j} c_{2}^{j}(0) \\
+\lambda^{j} D_{2}\left(c_{2}^{j}(0)\right) \frac{\partial c_{2}^{j}(y)}{\partial y} .
\end{gathered}
$$

В силу произвольности функций $w^{j}(x), z^{j}(x)$ из последних соотношений получим следующие прямые краевые задачи относительно функций $w^{j}(x)$ и $z^{j}(x)$ :

$$
\begin{gathered}
\frac{w^{j}(y)-c_{1}^{j-1}(y)}{\Delta t}-r^{j} \frac{d}{d y}\left(D_{1}\left(c_{1}^{j-1}(y)\right) \frac{d w^{j}(y)}{d y}\right) \\
-q^{j}(y) \frac{d w^{j}(y)}{d y}=0 \\
w^{j}(0)=0 \\
D_{1}\left(c_{1}^{j-1}(1)\right) \frac{\partial w^{j}(1)}{\partial y}+p^{j} w^{j}(1)=p^{j} c_{2}^{j}(0) \\
+\lambda^{j} D_{2}\left(c_{2}^{j}(0)\right) \frac{\partial c_{2}^{j}(0)}{\partial y}
\end{gathered}
$$




$$
\begin{aligned}
& \frac{z^{j}(y)}{\Delta t}-r^{j} \frac{d}{d y}\left(D_{1}\left(c_{1}^{j-1}(y)\right) \frac{d z^{j}(y)}{d y}\right) \\
& -q^{j}(y) \frac{d z^{j}(y)}{d y}=0, \\
& z^{j}(0)=1, \\
& D_{1}\left(c_{1}^{j-1}(1)\right) \frac{\partial z^{j}(1)}{\partial y}+p^{j} z^{j}(1)=0 .
\end{aligned}
$$

Подставляя (23) в функционал (22), будем иметь

$$
J\left(f^{j}\right)=\left[w^{j}(1)+f^{j} z^{j}(1)-c_{1}^{r, j}\right]^{2}+\gamma\left(f^{j}\right)^{2} .
$$

Используя необходимое условие минимума функционала, получим

$$
2\left\lfloor w^{j}(1)+f^{j} z^{j}(1)-c_{1}^{r, j}\right\rfloor z^{j}(1)+2 \gamma f^{j}=0 .
$$

Из последнего уравнения можно определить $f^{j}$

$$
f^{j}=\frac{\left[c_{1}^{r, j}-w^{j}(1)\right] z^{j}(1)}{\left[z^{j}(1)\right]^{2}+\gamma}, \quad j=\overline{1, m} .
$$

Таким образом, из полученных соотношений можно конструировать следующий вычислительный алгоритм для численного решения обратной задачи $(17)-(21)$ по определению $f^{j}, j=\overline{1, m}$ :

1) для фиксированного значения временного слоя $j$ определяются решения прямых краевых задач (24)-(26) и $(27)-(29)$, т. е. функции $w^{j}(y), z^{j}(y)$ в интервале $[0,1]$;

2) из соотношения (30) определяется $f^{j}-$ приближенное значение искомой функции $f(t)$ при $t=t_{j}$;

$3)$ по формуле (23) определяется $c_{1}^{j}(y)$, т. е. приближение к функции $c_{1}(y, t)$ при $t=t_{j}$;

при переходе на следующий временной слой описанная процедура вычислений снова повторяется.

Для численного решения задач $(24)-(26)$ и $(27)-(29)$ можно использовать метод конечных разностей. Дискретные аналоги задач $(24)-(26)$ и $(27)-(29)$ на сетке $\varpi$ можно представить в виде

$$
\begin{gathered}
\frac{w_{i}^{j}-c_{1, i}^{j-1}}{\Delta t}-\frac{r^{j}}{\Delta y}\left[D_{1, i+1 / 2}^{j-1} \frac{w_{i+1}^{j}-w_{i}^{j}}{\Delta y}-D_{1, i-1 / 2}^{j-1}\right. \\
\left.\times \frac{w_{i}^{j}-w_{i-1}^{j}}{\Delta y}\right]-q_{i}^{j} \frac{w_{i+1}^{j}-w_{i}^{j}}{\Delta y}=0, \quad i=\overline{1, n-1}, \\
w_{0}^{j}=0, \\
D_{1, n}^{j-1} \frac{w_{n}^{j}-w_{n-1}^{j}}{\Delta y}+p^{j} w_{n}^{j}=p^{j} c_{2,0}^{j}+\lambda^{j} D_{2,0}^{j} \frac{c_{2,1}^{j}-c_{2,0}^{j}}{\Delta y}, \\
\frac{z_{i}^{j}}{\Delta t}-\frac{r^{j}}{\Delta y}\left[D_{1, i+1 / 2}^{j-1} \frac{z_{i+1}^{j}-z_{i}^{j}}{\Delta y}-D_{1, i-1 / 2}^{j-1} \frac{z_{i}^{j}-z_{i-1}^{j}}{\Delta y}\right] \\
-q_{i}^{j} \frac{z_{i+1}^{j}-z_{i}^{j}}{\Delta y}=0, \quad i=\frac{1, n-1}{1,},
\end{gathered}
$$

$$
\begin{gathered}
z_{0}^{j}=1, \\
D_{1, n}^{j-1} \frac{z_{n}^{j}-z_{n-1}^{j}}{\Delta y}+p^{j} z_{n}^{j}=0, \\
j=\overline{1, m}
\end{gathered}
$$

где $w_{i}^{j} \approx w^{j}\left(y_{i}\right), D_{1, i \pm 1,2}^{j-1} \approx D_{1}\left(c_{1}^{j}\left(y_{i \pm 1 / 2}\right)\right), z_{i}^{j} \approx z^{j}\left(y_{i}\right)$.

Полученные разностные задачи (31)-(33) и (34)-(36) при каждом фиксированном значении $j=1,2, \ldots, m$ представляют собой систему линейных алгебраических уравнений с трехдиагональной матрицей и для решения этих систем можно использовать алгоритм Томаса.

Таким образом, предложенный численный метод позволяет в каждом временном слое последовательно определить распределение концентрации растворенного вещества во второй фазе, концентрацию на границе $x=0$ и распределение концентрации в первой фазе.

\section{Результаты численных расчетов}

Для проверки эффективности предложенного вычислительного алгоритма были проведены численные эксперименты для модельных задач. Схема численного эксперимента состоит в следующем. Для заданных функций $f(t), s(t)$ последовательно решаются прямые задачи $(7)-(10)$ и $(11)-(14)$. Найденная зависимость $c_{1}^{r}(t)=c_{1}(1, t)$ принимается за точные данные для численного решения обратной задачи по восстановлению $f(t)$. Первая серия расчетов выполнялась с использованием невозмущенных данных. Вторая серия расчетов проводилась при наложении на $c_{1}^{r}(t)$ некоторой функции, моделирующей погрешность входных данных

$$
\tilde{c}_{1}^{r}(t)=c_{1}^{r}(t)+\delta c_{1}^{r}(t) \sigma(t),
$$

где $\sigma(t)$ - случайный процесс, моделируемый с помощью датчика случайных чисел, $\delta$ - уровень погрешности.

Расчеты выполнялись на пространственно-временной разностной сетке с шагами $\Delta y=0.02 \mathrm{~m}, \Delta t=0.5$ и $2 \mathrm{~s}$. Результаты численного эксперимента, проведенного для случаев $s(t)=v t, D_{1}\left(c_{1}\right)=D_{0}\left(1+g_{1} c_{1}\right) \mathrm{m}^{2} / \mathrm{s}, D_{2}\left(c_{2}\right)=$ $=D_{0}\left(1+g_{2} c_{2}\right) \mathrm{m}^{2} / \mathrm{s}, \quad f(t)=0.6+0.2 \sin 2 t \mathrm{~kg} / \mathrm{kg}, \quad l=$ $=0.6 \mathrm{~m}, g_{1}=0.01, \phi_{1}(y)=0.4 \mathrm{~kg} / \mathrm{kg}, \phi_{2}(y)=0.2 \mathrm{~kg} / \mathrm{kg}$, $c_{2}^{r}(t)=0.3 \mathrm{~kg} / \mathrm{kg}, \quad \xi(t)=0.2 \mathrm{~kg} / \mathrm{kg}, \quad g_{2}=0.02, \quad v=$ $=0.002 \mathrm{~m} / \mathrm{s}, \quad D_{0}=5 \cdot 10^{-2} \mathrm{~m}^{-2} / \mathrm{s}$ при использовании невозмущенных и возмущенных входных данных, представлены в таблице.

Как показывают результаты численного эксперимента, при использовании невозмущенных входных данных искомая функция $f(t)$ восстанавливается с очень высокой точностью при всех расчетных сетках по времени (3-й и 4-й столбцы). При использовании невозмущенных входных данных для параметра регуляризации принять $\gamma=0$. 
Результаты численного эксперимента

\begin{tabular}{r|c|c|c|c|c}
\hline \multirow{2}{*}{$t, \mathrm{~s}$} & \multirow{2}{*}{$f^{t}$} & $\bar{f}$ & $\bar{f}$ & \multicolumn{2}{|c}{$\tilde{f}$} \\
\cline { 5 - 6 } & & $\Delta t=0.5 \mathrm{~s}$ & $\Delta t=2 \mathrm{~s}$ & $\begin{array}{c}\Delta t=0.5 \mathrm{~s} \\
\gamma=0.02\end{array}$ & $\begin{array}{c}\Delta t=2 \mathrm{~s} \\
\gamma=0.02\end{array}$ \\
\hline 2 & 0.449 & 0.449 & 0.449 & 0.444 & 0.410 \\
4 & 0.798 & 0.798 & 0.798 & 0.789 & 0.783 \\
6 & 0.493 & 0.493 & 0.493 & 0.494 & 0.504 \\
8 & 0.542 & 0.543 & 0.543 & 0.541 & 0.537 \\
10 & 0.783 & 0.784 & 0.783 & 0.781 & 0.778 \\
12 & 0.419 & 0.419 & 0.420 & 0.417 & 0.425 \\
14 & 0.654 & 0.658 & 0.654 & 0.655 & 0.651 \\
16 & 0.710 & 0.712 & 0.711 & 0.703 & 0.703 \\
18 & 0.402 & 0.403 & 0.402 & 0.416 & 0.403 \\
20 & 0.749 & 0.752 & 0.749 & 0.746 & 0.750
\end{tabular}

Примечание. $t$ - время, $f^{t}$ - точные значения функции $f(t)$, $\bar{f}$ - вычисленные значения $f(t)$ при невозмущенных данных, $\tilde{f}-$ вычисленные значения $f(t)$ при возмущенных данных.

При использовании возмущенных входных данных, в которых погрешность имеет флуктуационный характер, искомая функция $f(t)$ восстанавливается с определенной погрешностью. Для возмущения входных данных в качестве уровня погрешности использовались $\delta=0.05$. Следует отметить, что значение параметра регуляризации должно быть согласовано с уровнем погрешности во входных данных. При проведении численных расчетов значения этого параметра определялись по невязке путем численного экспериментирования.

Анализ результатов численного эксперимента показывает, что предложенный вычислительный алгоритм можно применять при изучении диффузионных фазовых превращений в твердых телах.

\section{Список литературы}

[1] Любов Б.Я. Кинетическая теория фазовых превращений. М.: Металлургия, 1969. 264 с.

[2] Любов Б.Я. Диффузионные процессы в неоднородных твердых средах. М.: Наука, 1981. 296 с.

[3] Мерер X. Диффузия в твердых телах. Долгопрудный: Изд. Дом „Интеллект“, 2011. 536 с.

[4] Бокштейн Б.С. Диффузия в металлах. М.: Металлургия, 1978. 248 c.

[5] Рубинштейн Л.И. Проблема Стефана. Рига: Звайгзне, 1967. $232 \mathrm{c.}$

[6] Самарский А.А., Вабищевич П.Н. Вычислительная теплопередача. М.: Едиториал, 2003. 784 с.

[7] Алифанов О.М., Артюхин Е.А., Румянщев С.В. Экстремальные методы решения некорректных задач. М.: Наука, 1988. 288 c.

[8] Самарский А.А., Вабищевич П.Н. Численные методы решения обратных задач математической физики. М.: Изд-во ЛКИ, 2009. 480 с.

[9] Borukhov V.T., Zayats G.M. // Intern. J. Heat and Mass Transfer. 2015. Vol. 91. P. 1106-1113.

[10] Гамзаев Х.М. // Вестник ТомГУ. Математика и Механика. 2017. № 50. C. $67-78$. 